\title{
Ubuntu ethics: Putting your culture first in the hospital workplace
}

\begin{abstract}
Ubuntu is very difficult to render into a Western language. It speaks of the very essence of being human ... you are generous, you are hospitable, you are friendly and caring and compassionate. You share what you have. It is to say, 'My humanity is inextricably bound up in yours.' We belong in a bundle of life. (Desmond Tutu)
\end{abstract}

To the Editor: Esprit de corps, a French phase referring to morale and dictating 'a spirit of togetherness', should govern how we serve patients together. Closer to home, and far more profound and allencompassing, the Nguni word ubuntu can be defined as 'generosity, respect for man irrespective of position' or 'I am because we are'. It is easy to imagine how one could lose this spirit of warm embrace towards others when talking over a phone in a laboratory. In fact, any doctor sometimes forgets to embrace the spirit of ubuntu while working in a stressful environment. We are focused on individual ethics, we function in silos and we forget our African morals of communitarianism. We morally justify how to socially interact with different patients, at times neglecting our rich cultural diversity and differences. These cultural differences are recognised in bioethical guidelines, but the solutions remain Western-dominated.

Various research articles have looked at ubuntu, with some focusing on the analytical processes required to illustrate how a social theory and political ideal can be developed out of their constitutive elements to recommend a coherent conceptual framework in South African (SA) public policy. ${ }^{[1]}$ Others have applied the principle to conflicting African public health and medical problems, as well as to solving legal matters in the SA context. ${ }^{[2,3]}$

But ubuntu involves much more than just looking after the best interests of the community. It is about having compassion, and being friendly to a patient and his or her family, respecting their cultures, wisdom and beliefs. It builds on the core foundation that we as a multidisciplinary team should be kind and generous towards one another. It unites us in the workplace, and entrenches the virtues we find morally valuable and desirable. It is more than just a habit of doing things; it is a way of life, our 'Africanness'. It was
Nelson Mandela who said: 'In Africa, there is a concept known as ubuntu - the profound sense that we are human only through the humanity of others, that if we are to accomplish anything in this world, it will in equal measure be due to the work and achievement of others.'

It is through teamwork and mutual respect among different disciplines, from pathology to medicine, and thus biomedical sciences to bedside patient enablement, that we can shift our application of ethics to the everyday values of the African people and learn from one another to embrace the concept 'I am because we are.' So the next time you phone the laboratory, require a specialist consult or are simply working with a junior colleague or a nurse, consider the spirit of ubuntu and respect African culture in our hospitals.

Acknowledgements. I would like to acknowledge Prof. A Brink, head of the Division of Medical Microbiology, Department of Pathology, Faculty of Health Sciences, University of Cape Town, for providing insight into the article.

\section{J Opperman}

Division of Medical Microbiology, National Health Laboratory Service, University of Cape Town, and Groote Schuur Hospital, Cape Town, South Africa Stefanopperman1@gmail.com

1. Nkondo GM. Ubuntu as public policy in South Africa: A conceptual framework Int J Afr Renaissance Stud - Multi- Inter- Transdisciplinarity 2007;2(1):88-100. https://doi.org/10.1080/18186870701384202

2. Himonga C, Taylor M, Pope A. Reflections on judicial views of Ubuntu. Potchefstroom Electr Law J 2013;16(5):369-427. http://doi.org/10.4314/pelj. v16i5.8

3. Sambala EZ, Cooper S, Manderson L. Ubuntu as a framework for ethical decisionmaking in Africa: Responding to epidemics. J Ethics Behavior 2019;0(0):1-13. https://doi.org/10.1080/10508422.2019.1583565

S Afr J Bioethics Law 2019;12(1):3. DOI:10.7196/SAJBL.2019.v12i1.682 